\title{
Prefrontal White Matter Structure Mediates the Influence of GADI on Working Memory
}

\author{
Tristram A Lett ${ }^{1,2}$, James L Kennedy ${ }^{2,3,4}$, Natasha Radhu ${ }^{2,4}$, Luis G Dominguez ${ }^{2}$, M Mallar Chakravarty ${ }^{5,6}$, \\ Arash Nazeri' ${ }^{2,3}$, Faranak Farzan ${ }^{2,4}$, Henrik Walter', Andreas Heinz', Benoit H Mulsant ${ }^{2,3,4}$, \\ Zafiris J Daskalakis ${ }^{2,3,4,7}$ and Aristotle N Voineskos ${ }^{*, 2,3,4,7}$
}

'Department of Psychiatry and Psychotherapy, Charité Universitätsmedizin, Berlin, Germany; ${ }^{2}$ Campbell Family Mental Health Institute, Centre for Addiction and Mental Health, Toronto, ON, Canada; ${ }^{3}$ Department of Psychiatry, University of Toronto, Toronto, ON, Canada; ${ }^{4}$ Institute of Medical Science, University of Toronto, Toronto, ON, Canada; ${ }^{5}$ Cerebral Imaging Centre, Douglas Hospital Mental Health University Institute, Verdun, QC, Canada; ${ }^{6}$ Departments of Psychiatry and Biomedical Engineering, McGill University, Montreal, QC, Canada

\begin{abstract}
The glutamic acid decarboxylase I (GADI) gene is a major determinant of $\boldsymbol{\gamma}$-aminobutyric acid (GABA), the most abundant inhibitory neurotransmitter modulating local neuronal circuitry. GABAergic dysfunction and expression of GAD / have been implicated in the pathophysiology of schizophrenia, and in working memory impairment. We examined the influence of the functional GAD/ rs3749034 variant on white matter fractional anisotropy (FA), cortical thickness, and working memory performance in schizophrenia patients and healthy controls $(N=197)$. Using transcranial magnetic stimulation with electroencephalography (TMS-EEG), we subsequently examined the effect of rs3749034 on long-interval cortical inhibition (LICI) in the dorsolateral prefrontal cortex (DLPFC) in schizophrenia patients and healthy controls $(N=66)$. We found that the rs3749034 T-allele carrier risk group had lower voxel-wise FA in the prefrontal cortex region $\left(P_{\text {FWE-corrected }}<0.05\right)$ but not cortical thickness. Mixed-model regression revealed a significant effect on attentional processing and working memory across four performance measures $\left(F_{1,182}=11.5, P=8 \times 10^{-4}\right)$. FA in the prefrontal cortex was associated with digit-span performance. Voxel-wise mediation analysis revealed that the effect GADI on poorer digit-span performance statistically

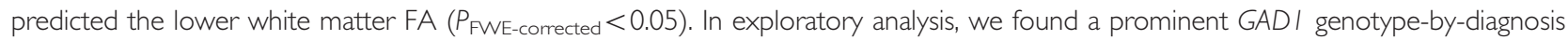
interaction on DLPFC LICl $\left(F_{1,56}=14.3, P=4.1 \times 10^{-4}\right)$. Our findings converge on variation in GAD/ gene predicting a susceptibility mechanism that affects white matter FA, GABAergic inhibitory neurotransmission in the DLPFC, and working memory performance. Furthermore, via voxel mediation of FA and TMS-EEG intervention, we provide evidence for a potentially causal mechanism through which aberrant DLPFC GABA signaling may contribute to working memory dysfunction.

Neuropsychopharmacology (2016) 4I, 2224-223I; doi: I0.1038/npp.20I6.I4; published online 24 February 2016
\end{abstract}

\section{INTRODUCTION}

Working memory dysfunction is a central feature of schizophrenia and other psychiatric disorders. In patients with schizophrenia, working memory deficits are associated with dysfunction of dorsolateral prefrontal cortex (DLPFC) connectivity, and disruption of neurotransmitter input such as $\gamma$-aminobutyric acid (GABA) inhibitory neurotransmission (Lewis et al, 2012). There is a genetic contribution to working memory dysfunction. Unaffected cotwins of schizophrenia patients perform significantly worse than controls on spatial and verbal working memory tasks (Pirkola et al,

\footnotetext{
*Correspondence: Dr AN Voineskos, Kimel Family Translational Imaging-Genetics Laboratory, Research Imaging Centre, Campbell Family Mental Health Institute, Centre for Addiction and Mental Health, 250 College Street, Toronto, ON, Canada M5TIR8, Tel: + I 4I6535850। ext 4378,E-mail: Aristotle.Voineskos@camh.ca ${ }^{7}$ Co-senior author

Received 10 October 2015; revised 9 January 2016; accepted 25 January 2016; accepted article preview online 29 January 2016
}

2005). For example, the letter-number-sequencing task (LNS; a measure of working memory) has been identified as an endophenotype of schizophrenia with a moderately high heritability $\left(h^{2}=0.39\right)$ (Greenwood et al, 2007).

Heritability studies have also demonstrated that prefrontal and temporal gray matter volumes are strongly influenced by genetic factors in comparison with other regions of the cortex (Thompson et al, 2001). Diffusion tensor imaging heritability studies generally reported the greatest impact of genetic factors on fractional anisotropy (FA) in cingulate gyrus, uncinate fasciculi, and the corpus callosum (Kochunov et al, 2014). Taken together, this suggests that working memory functioning is mediated, in part, by genetic factors driving brain structure and function. However, it could be argued that imaging-genetic studies of working memory are intrinsically correlational, showing only associations among genetic variants, brain structure, and cognitive performance (Kanai and Rees, 2011). To date, few studies have examined if the genetic contribution to working 
memory is mediated by brain structure (Aarts et al, 2010; Green et al, 2013; Klumpers et al, 2014).

One method that may resolve the causal relationship among genes, brain structure, and working memory performance is transcranial magnetic stimulation with electroencephalography (TMS-EEG). Combined TMS-EEG applied over the DLPFC can be used to measure the effects of GABAergic inhibitory neurotransmission on neural oscillations and synchrony that are thought to be integral to higher order cognitive function including working memory (Daskalakis et al, 2008; Lett et al, 2014; McClintock et al, 2011). Paired-pulse cortical stimulation can elicit cortical inhibition or facilitation that is suggested to underlie neurotransmission at the synapse. In particular, longinterval cortical inhibition (LICI) has been linked to metabotropic $\mathrm{GABA}_{\mathrm{B}}$ receptor transmission (Deisz, 1999; McDonnell et al, 2006). DLPFC LICI has been correlated with performance on the N-back $(r=0.63)$, LNS $(r=0.68)$, and Sternberg (accuracy: $r=0.58$; reaction time: $r=0.48$ ) working memory tasks in healthy controls (Daskalakis et al, 2008; Hoppenbrouwers et al, 2012; Rogasch et al, 2014). Therefore, DLPFC LICI may represent an important mechanism linking GABAergic inhibitory neurotransmission and working memory performance.

Convergent evidence suggests a compelling role for the glutamate decarboxylase 1 (GAD1) gene in GABAergic neurotransmission as it relates to working memory. GAD1 codes for the glutamic acid decarboxylase $\left(\mathrm{GAD}_{67}\right)$ enzyme that metabolizes glutamate to GABA and is responsible for the production of the majority of GABA in the brain (Lewis et al, 2005), although activity-induced GABA synthesis has been shown to be induced by $\mathrm{GAD}_{65}$ (Patel et al, 2006). Downregulation of $\mathrm{GAD}_{67}$ in the parvalbumin-positive interneurons of the DLFPC is a well-replicated post-mortem finding in schizophrenia (Torrey et al, 2005). In the mouse prefrontal cortex, $\mathrm{GAD}_{67}$ deficits in the parvalbumin interneurons have been causally linked to inhibitory transmission and network disinhibition (Lazarus et al, 2013). Given the linkage disequilibrium structure of GAD1, the rs3749034 single-nucleotide polymorphism (SNP) covers a large proportion of the variance in the promoter/5' region; rs3749034 has been associated with the downregulation of $\mathrm{GAD}_{67}$ in the DLPFC as well as with the progressive switch from GAD25 to GAD67 expression and KCC2 to NKCC1 expression (Hyde et al, 2011; Straub et al, 2007). This switch is thought to be the hallmark of the shift from GABA excitatory function to inhibitory function underlying synaptic wiring and refinement of local neuronal circuits (Ben-Ari et al, 2012). Furthermore, a 5'-promoter SNP in linkage disequilibrium with rs3749034 is associated with variation in working memory performance and DLPFC function in vivo (Straub et al, 2007). Therefore, the GAD1 rs3749034 SNP may reflect changes in cortical connectivity and local inhibitory circuits that are involved in working memory performance.

In the present study, we set out to provide convergent evidence that the relationship between GAD1 and working memory performance may be mediated by brain structural connectivity and DLPFC inhibitory neurotransmission. As GAD1 is expressed ubiquitously across the cortex, we examined whole-brain associations of cortical thickness and white matter FA connecting these cortical regions. Further, it is unclear if GAD1 would predict specific working memory domains or general prefrontal executive functioning. Therefore, we tested associations among the GAD1 promoter variant rs3749034, and neurocognitive tasks spanning attention in addition to verbal and non-verbal components of working memory. Using LICI, we explored the association among the rs3749034 SNP and DLPFC cortical inhibition.

\section{MATERIALS AND METHODS}

\section{Participants}

Two independent samples were examined. Participants for both the imaging-genetic sample and the TMS-EEG sample were recruited under separate study protocols approved by the Center for Addiction and Mental Health (CAMH) in Toronto, Canada in accordance with the Declaration of Helsinki. All participants were identified as Caucasian based on self-reported ethnicity of three out of four grandparents. They were administered the Structured Clinical Interview for DSM-IV Disorders (First et al, 1995), and were interviewed by a psychiatrist to ensure diagnostic accuracy. Individuals with previous head trauma with loss of consciousness, neurological disorders, current or past substance dependence, and a history of a primary psychotic disorder in first-degree relatives were excluded.

For the imaging-genetic sample, all clinical assessments occurred at CAMH, while T1-weighted and diffusionweighted magnetic resonance imaging (DW-MRI) scans were performed at a nearby general hospital. Eighty patients with a diagnosis of schizophrenia or schizoaffective disorder and 115 healthy control subjects in this sample completed all imaging and genetic protocols; three healthy controls did not complete DW-MRI protocols. Seventy patients and 121 healthy controls completed cognitive testing, and genetic protocols. IQ was measured using the Wechsler Test for Adult Reading (WTAR) (Holdnack, 2001) and all participants were screened with the Mini Mental Status Exam for dementia (Folstein et al, 1975) and a urine toxicology screen. The Hand Dominance Questionnaire was used to examine handedness. Comorbid physical illness burden was measured by administration of the Clinical Information Rating Scale for Geriatrics (Miller et al, 1992). All subjects underwent a battery of cognitive test that have been described previously (Voineskos et al, 2012). See Supplementary 1 for detail on the assessment of working memory.

For the TMS-EEG sample, all clinical assessments and TMSEEG recordings occurred at CAMH. The sample has been described previously (Radhu et al, 2015). For the purpose of this study, only participants of Caucasian ethnicity were included in the analysis, resulting in 33 healthy controls and 23 patients with a diagnosis of schizophrenia or schizoaffective disorder with completed TMS-EEG and genetic protocols. Exclusion criteria established by international safety standards for TMS were followed (Rossi et al, 2009). The TMS adult safety screen was administered to all subjects (Keel et al, 2001).

\section{Genetics}

Genotyping of the rs3749034 variant (GAD1) was performed using a standard ABI (Applied Biosystems) 5' nuclease Taqman assay-on-demand protocol in a total volume of 
$10 \mu$ l. Postamplification products were analyzed on the $\mathrm{ABI}$ 7500 Sequence Detection System (ABI, Foster City, CA) and genotype calls were performed manually. Results were verified independently by laboratory personnel blind to demographic and phenotypic information. Genotyping accuracy was assessed by repeating $10 \%$ of the sample with $100 \%$ accordance in all genotype calls.

\section{Image Acquisition}

High-resolution axial inversion recovery-prepared spoiled gradient recall $\mathrm{MR}$ images were acquired using a 1.5-T GE Echospeed System (General Electric Medical Systems, Milwaukee, WI; echo time (TE): $5.3 \mathrm{~ms}$, repetition time (TR): $12.3 \mathrm{~ms}$, time to inversion: $300 \mathrm{~ms}$, flip angle 20 , number of excitations $=1 ; 124$ contiguous images, $1.5 \mathrm{~mm}$ thickness). For DW-MRI acquisition, a single-shot spin-echo planar sequence was used with diffusion gradients applied in 23 noncollinear directions, $b=1000 \mathrm{~s} / \mathrm{mm}^{2}$, and two $b=0$ images. Fifty-seven slices were acquired for whole-brain coverage oblique to the axial plane $(2.6 \mathrm{~mm}$ isotropic voxels; field of view was $330 \mathrm{~mm}, 128 \times 128 \mathrm{~mm}^{2}$ acquisition matrix; $\mathrm{TE}=85.5 \mathrm{~ms}, \mathrm{TR}=15000 \mathrm{~ms}$; the sequence was repeated three times to improve signal-to-noise ratio). Quality control for each raw diffusion-weighted scan was conducted manually by an experienced operator before inclusion of the images into the research study. Each scan was visually inspected for artifacts or motion including: horizontal stripes, checker effects, inter- and intraslice intensity. Cortical thickness mapping and tract-based spatial statistics are explained in detail in Supplementary 1, Methods and Materials.

\section{Cluster-Based Measure of Long-Interval Cortical Inhibition}

LICI was assessed among all electrodes at frequencies ranging from 1 to $50 \mathrm{~Hz}$. Cluster-based analysis has been previously described in detail in this sample (Garcia Dominguez et al, 2014; Radhu et al, 2015), and based on analyses described by Maris and Oostenveld (2007). Clusters are defined in a time-frequency-space domain, a fourdimensional entity, as space is the two dimensional grid of electrodes plus one dimension for time and another for frequency. Cortical inhibition was assessed at every timefrequency-spatial value using a one-tailed, paired $t$-test testing if the single-pulse condition was significantly greater than the paired-pulse stimulation. The significant threshold was set at $\alpha<0.01$ after 10000 permutations of trials from the two conditions, paired and single pulse. The number of significant voxels within the biggest cluster of inhibition was calculated for every subject, and it was determined as the degree of LICI after stimulation to the DLPFC. This measure of DLPFC LICI was left-skewed, and therefore we applied a $\log$ transformation. See Supplementary 1 for detail on localization of the DLPFC for TMS-EEG, LICI assessment, EEG recording, and processing.

\section{Statistics}

Given the low frequency of the TT genotype, these cases were grouped with CT genotypes and referred to as 'T-allele carriers' for all statistical tests (Supplementary Table S1).
Analyses were performed examining the relationship between GAD1 genotype group (C allele homozygotes or T-allele carriers) for cognitive performance, brain morphology, and DLPFC LICI. Adherence to Hardy-Weinberg equilibrium and case-control allelic frequency association (using $\chi^{2}$ ) were determined using Haploview 4.2.

Differences in demographic characteristics between healthy controls and schizophrenia patients were assessed by independent $t$-tests for continuous variables and $\chi^{2}$ tests for count variables using SPSS (Version 21). For cortical thickness and tract-based spatial statistics (TBSS) analyses, we examined a diagnosis by GAD1 genotype interaction, and main effects of diagnosis and genotype on the FA skeleton with age and sex as covariates. A false discovery rate (FDR) correction of $q=0.05$ was applied for cortical thickness, and a family-wise error (FWE) rate of $5 \%$ for white matter FA comparisons. Multiple comparison corrections were performed within each analytic imaging modality.

To determine potential confounders of working memory, we first evaluated if age, sex, IQ, and three measures of education (participant, father, mother) were predictors of task performance (LNS, digit-span) in healthy controls or schizophrenia patients via the linear mixed model. Task performance scores were the within-subject variable. GAD1 genotype, diagnosis, and their interaction were the betweensubject factors. Follow-up analysis was carried out using the general linear model (GLM). Covariates of no interests were only included in our model if they were below a significance threshold of $P=0.1$. The influence of GAD1 as a predictor of cognitive performance, diagnosis, and their interaction was then analyzed via the GLM with our covariates of no interest. Four GLMs were performed with LNS score, digit-span, Stroop (time/item), and Stroop ratio. Associations were deemed significant at $P<0.05$ after Bonferroni correction for four comparisons.

\section{Voxel-Wise Mediation Analysis}

Mediation was assessed at each FA voxel from the TBSS skeleton. GAD1 rs3749034 genotype was the independent variable, neurocognitive performance was the dependent variable, and each FA voxel was the mediator. Age, sex, and diagnosis were included as covariates. Significant mediation was assessed via the Sobel Z-statistic at each voxel creating a TBSS skeleton map of Sobel Z-statistics, which then underwent threshold-free clustering enhancement. Significant after family-wise correction was assessed by maximum whole skeleton threshold-free clustering enhancement value from 10000 permutations (randomization). See Supplementary 1, Methods and Materials for a detailed description of the voxel-wise mediation analysis.

\section{RESULTS}

\section{Participants}

There was no significant deviation from Hardy-Weinberg equilibrium for the rs3749034 genotype $(P=1$; Supplementary Table S1). The rs3749034 minor allele frequency of the healthy controls $(0.22)$ was similar to Hapmap CEU population (0.18; Hapmap Build No. 27) (Gibbs et al, 2003). The frequency of the rs3749034 $\mathrm{T}$ allele 
Table I Demographics of Imaging-Genetic Sample

\begin{tabular}{|c|c|c|c|c|c|c|}
\hline & \multicolumn{2}{|c|}{$\begin{array}{l}\text { Controls } \\
(N=\text { I I5) }\end{array}$} & \multicolumn{2}{|c|}{$\begin{array}{l}\text { Schizophrenia } \\
\qquad(N=80)\end{array}$} & \multirow[t]{2}{*}{$t_{193}$} & \multirow[t]{2}{*}{$P$-value } \\
\hline & Mean & SD & Mean & SD & & \\
\hline Age (years) & 46.79 & 19.32 & 45.49 & 16.00 & 0.49 & 0.63 \\
\hline IQ (WTAR score) & | 17.92 & 8.11 & 110.64 & 15.16 & 4.24 & $<0.05$ \\
\hline Education (years) & 15.32 & 1.95 & 13.25 & 2.64 & 6.27 & $<0.05$ \\
\hline \multirow{2}{*}{$\begin{array}{l}\text { Highest level of } \\
\text { education (parent) }\end{array}$} & 5.16 & 1.81 & 4.18 & 2.26 & 3.38 & $<0.05$ \\
\hline & Count & Frequency & Count & Frequency & $\chi^{2}$ & $P$ \\
\hline Handedness (R) & 105 & $92.1 \%$ & 71 & $89.9 \%$ & 0.23 & 0.59 \\
\hline $\operatorname{Sex}(M)$ & 61 & $53.0 \%$ & 48 & $60.0 \%$ & 0.93 & 0.38 \\
\hline
\end{tabular}

was significantly greater in schizophrenia patients (0.29) than in controls $\left(0.22, \chi^{2}=3.8, P=0.05\right)$, and the association is in the same direction as the most recent findings from the Psychiatric Genomics Consortium (T risk allele; $P=0.007$ ) (Schizophrenia Working Group of the Psychiatric Genomics Consortium, 2014). For both samples, age, sex, and handedness were not significantly different between schizophrenia patients and healthy controls $(P>0.05)$. In the imaging-genetic sample, patients had lower IQ, education, and highest level of education of either parent $(P<0.05$; Table 1).

\section{Association between GAD1 and Brain Structure}

We found no effect of genotype or genotype-by-diagnosis interaction at any cortical thickness vertex after covarying for age and using FDR correction of 5\%. We found a significant main effect in which T-allele risk carries had lower FA, predominately in the prefrontal cortex including the genu of the corpus callosum $\left(P_{\text {FWE-corrected }}<0.05\right.$; Figure 1 and Supplementary Figure S1). There was no significant GAD1 genotype-by-diagnosis interaction on FA after correcting for FWE.

\section{Association between $G A D 1$ and Neurocognitive Performance}

We included age and IQ as covariates of no interest in our analyses as they were significantly associated with our working memory outcome measures (digit-span and LNS) in schizophrenia patients and healthy controls $(P<0.05)$. Sex and education were not associated with digit-span forward or LNS performance in either patients or controls $(P>0.1)$; therefore, they were not included in as covariates. In a linear mixed model, we included performance of our four cognitive measures (digit-span forward, LNS, Stroop time/item, Stroop ratio) as within-subject variables because of the correlation among these performance scores in healthy controls and schizophrenia patients (Supplementary Table S3).

There was a significant between-subject effect of GAD1 genotype $\left(\mathrm{F}_{1,182}=11.5, P=8 \times 10^{-4}\right)$, but no significant genotype-by-diagnosis interaction $\left(\mathrm{F}_{1,182}=2.3, \quad P=0.13\right)$. Further, there was a significant within-subject effect (task-

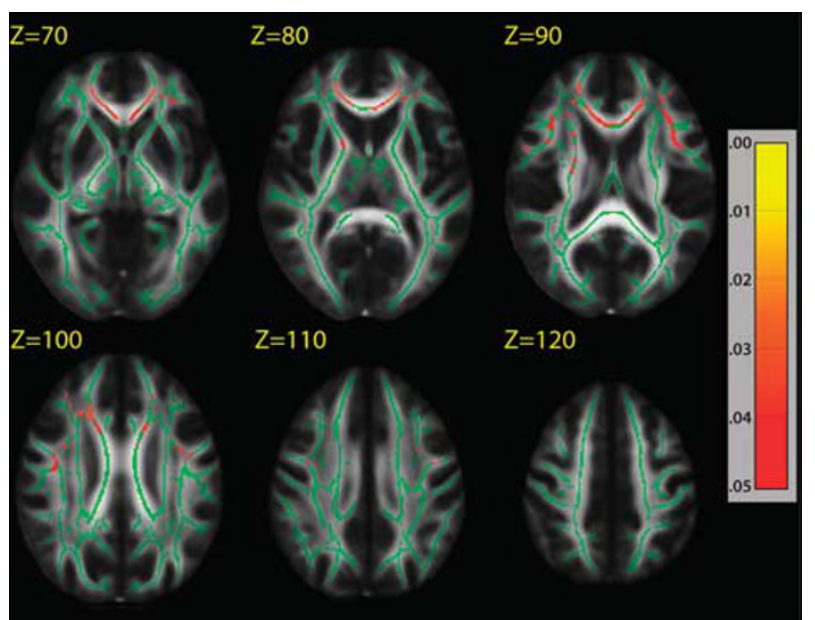

Figure I Glutamic acid decarboxylase I (GAD I) rs3749034 risk T-allele predicts lower tract-based spatial statistics (TBSS) skeleton white matter fractional anisotropy (FA) in healthy controls $(N=\mid 15)$ and patients with schizophrenia $(N=80)$. There was a significant main effect of GADI genotype on prefrontal FA, and no significant GAD / genotype-by-diagnosis interaction. Areas in red correspond to $P<0.05$ after correction for familywise error. Green represents the mean FA skeleton overlaid on the FMRIB58_FA standard space image.

by-genotype) association with GAD1 genotype $(P=0.03)$. Follow-up analyses in each task revealed a significant effect of $G A D 1$ on digit-span forward performance $\left(\mathrm{F}_{1,188}=7=8.0\right.$, $P=0.005)$ and a nominally significant effect on the LNS task performance $\left(\mathrm{F}_{1,188}=5.0, P=0.026\right)$, after covarying for subject age and IQ. There also was a significant association with Stroop ratio $\left(\mathrm{F}_{1,188}=7.0, P=0.009\right)$, but not time per item. No significant GAD1 genotype-by-diagnosis interaction was observed for any cognitive task (Table 2 and Supplementary Figure S2).

\section{Voxel-Wise Mediation Analysis}

As there was a significant association between main effect of $G A D 1$ risk genotype and white matter FA as well as working memory, we used voxel-wise mediation analysis across all subjects. For mediation to occur, we first needed to establish if white matter FA predicted working memory performance (corrected for IQ). Voxel-wise analysis of the TBSS skeleton revealed that higher FA was associated with better digit-span forward performance after covarying for the effect of age, particularly in the corpus callosum and the right superior longitudinal fasciculus $\left(P_{\text {FWE-corrected }}<0.05\right.$; Supplementary Figure S3). FA did not predict LNS performance or Stroop ratio after correcting for family-wise error; therefore, we did not perform any mediation analyses for these tasks. White matter skeleton FA mediated the association between GAD1 risk genotype and digit-span performance, particularly in the prefrontal and right inferior parietal regions, suggesting that lower FA in this region is statistically causing poorer digit-span performance $\left(P_{\text {FWE-corrected }}<0.05\right.$; Figure 2$)$.

\section{Long-Interval Cortical Inhibition}

In the TMS-EEG sample, GAD1 genotype and diagnosis were not associated with DLPFC LICI cluster size (GAD1 
Table 2 The Association Between Working-Memory Related Tasks and GAD/ rs3749034 Genotype, Diagnosis, and their Interaction

\begin{tabular}{|c|c|c|c|c|c|c|}
\hline \multirow[t]{2}{*}{ Working memory task } & \multicolumn{2}{|c|}{ GADI } & \multicolumn{2}{|c|}{ Diagnosis } & \multicolumn{2}{|c|}{ GADI $\times$ diagnosi } \\
\hline & $\mathbf{F}_{1,188}$ & $P$-value & $F_{1,188}$ & $P$-value & $\mathbf{F}_{1,188}$ & $P$-value \\
\hline Letter-number span & 5.03 & 0.026 & 18.04 & $<0.0001$ & 2.89 & 0.091 \\
\hline Digit span & 7.97 & 0.005 & 5.06 & 0.026 & 0.02 & 0.89 \\
\hline Stroop (time/item) & 1.17 & 0.28 & 24.41 & $<0.000 \mid$ & 0.17 & 0.68 \\
\hline Stroop ratio & 7.03 & 0.009 & 12.50 & 0.001 & 2.50 & 0.12 \\
\hline
\end{tabular}

Covariates in the model include age (46.3 years), and Wechsler Test of Adult Reading (WTAR) performance (| | 5.1$)$.

genotype: $\mathrm{F}_{1,56}=0.6, P=0.43$; diagnosis: $\left.\mathrm{F}_{1,56}=1.7, P=0.20\right)$; however, we found a prominent GAD1 genotypeby-diagnosis interaction explaining $22 \%$ of the variance in LICI DLPFC cluster size $\left(R^{2}=0.22, \quad \mathrm{~F}_{1,56}=13.5\right.$, $P=4.2 \times 10^{-4}$; Figure 3$)$. Given the significant genotypeby-diagnosis interaction, we examined the association between GAD1 and LICI cluster size separately in healthy controls and patients with schizophrenia. In healthy controls, GAD1 risk genotype predicted greater DLPFC LICI cluster size $\left(R^{2}=0.25, \mathrm{~F}_{1,33}=10.1, P=0.003\right)$. In contrast, GAD1 risk genotype was associated with lower DLPFC LICI cluster size in patients with schizophrenia $\left(R^{2}=0.19, \quad \mathrm{~F}_{1,23}=5.4\right.$, $P=0.04)$.

\section{DISCUSSION}

We have provided evidence that the GAD1 rs3749034 risk variant is associated with lower white matter FA that may be related to poor working memory performance and frontal executive functioning that is independent of diagnosis. Carriers of the GAD1 risk allele had poorer working memory, and lower white matter FA in the prefrontal cortices. Furthermore, our exploratory analysis indicates that the GAD1 risk variant differentially predicts DLPFC LICI depending on diagnosis. Last, white matter FA positively correlates with digit-span performance independent of diagnosis, and we could statistically infer a causal relationship via white matter FA mediates the effect on GAD1 on digit-span performance.

To the best of our knowledge, our study is the first to examine the effect of rs3749034 genotype on white matter FA. Our finding that T-allele risk carriers predicted lower FA, predominately in the genu of the corpus callosum, is consistent with the literature on top-down modulation of posterior brain regions during inhibition and attention tasks (Hopfinger et al, 2000). The T allele was also associated with worse performance on both the working memory tasks and the Stroop reaction time. This may suggest that the effect of $G A D 1$ genotype may be acting on general frontal executive function rather than working memory specifically. This is bolstered by the association of genotype with prefrontal FA, and is line with the concept that genetic variation can delineate brain structure or function that is common among multiple cognitive functions. Furthermore, we found the effect of rs3749034 on FA and working memory performance was consistent across diagnostic groups. This suggests that

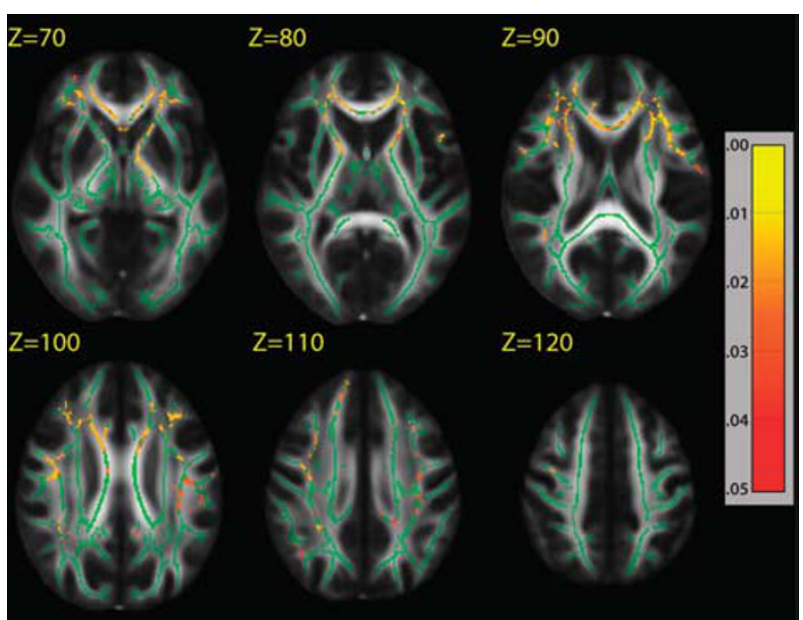

Figure 2 Skeletal white matter fractional anisotropy (FA) regions that mediate the effect of glutamic acid decarboxylase I (GADI) rs3749034 on digit-span forward performance. Significant $P$-values indicated broad areas of the white matter skeleton FA that mediated the effect of GAD / risk T-allele risk genotype on poor digit-span performance. We created a Z-statistic from voxel-wise Sobel tests for mediation based on the $\beta$-coefficients and standard error from (i) GADI regressed on tract-based spatial statistics (TBSS) skeleton FA (Figure I), and (ii) TBSS skeleton FA regressed on digitspan performance (Supplementary Figure S2). The $z$-values then underwent threshold free clustering enhancement, and $P$-values are derived using permutation testing $(N=10000)$. Areas in red correspond to $P<0.05$ after correction for family-wise error. Green represents the mean FA skeleton overlaid on the FMRIB58_FA standard space image.

genotypic variation in GAD1 acts as a modifier of brain structure and related cognitive function. GAD1 may explain some of the heterogeneity of brain structure and cognitive function reported within schizophrenia, despite the potential confounders within disease. Although our vertex-wise analysis did not replicate previous association between the GAD1 risk variant and the left parahippocampus (Brauns et al, 2013), our exploratory analysis revealed that the GAD1 risk variant may be associated with temporal lobe cortical thickness, although the effects may be modest. Replication is necessary to confirm the association.

This study is also the first to examine the effect of genetic variation on DLPFC LICI. Our exploratory TMS-EEG analysis yielded an unexpected result. In healthy controls, GAD1 T-allele carriers had greater LICI cluster size in contrast to schizophrenia patients in which T-allele carriers had lower cluster size. One speculative explanation could be that in healthy controls, T-allele carriers have less efficient LICI and need to incorporate more cortical areas to achieve the same inhibition. In contrast, schizophrenia patients may have reduced prefrontal connectivity, and the T-allele carriers are unable to elicit an LICI response to wider area. The link between disrupted GABA synthesis and $G_{A B A}$ receptor function, which is primarily measured by LICI, is not completely elucidated yet. Furthermore, the association among GAD1 genotype and LICI may be reliant on other neurotransmitter systems disrupted in schizophrenia. $\mathrm{GABA}_{\mathrm{A}}$ receptor neurotransmission generates a tonic conductance, in addition to phasic activations, suggesting that LICI may also index efficient $\mathrm{GABA}_{\mathrm{A}}$ receptor functioning (Farrant and Nusser, 2005). Additionally, 


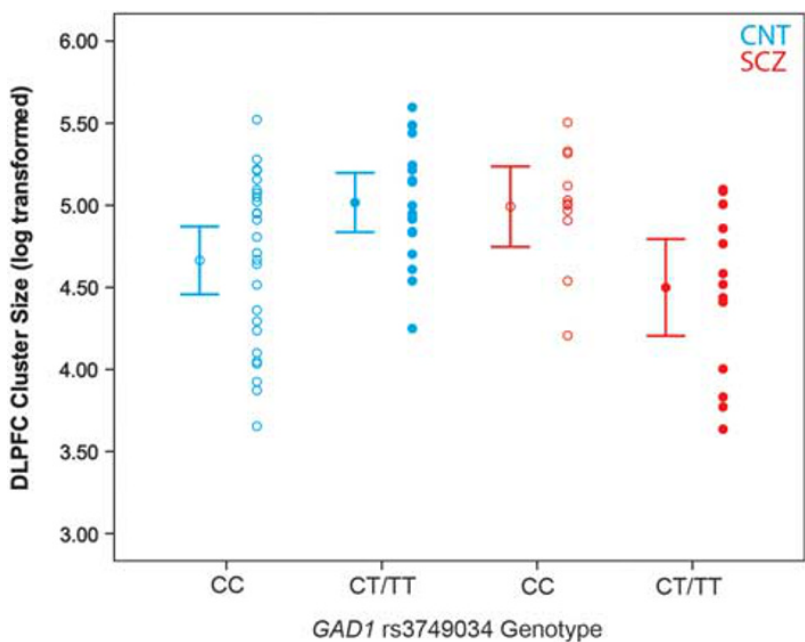

Figure 3 The effect of glutamic acid decarboxylase I (GAD I) rs3749034 genotype on long-interval cortical inhibition (LICl) cluster size in healthy control $(n=33)$ and patients with schizophrenia $(n=23)$ undergoing transcranial magnetic stimulation with electroencephalography (TMS-EEG) stimulation to the dorsolateral prefrontal cortex (DLPFC). Blue and red represent healthy controls and schizophrenia patients, respectively. Hollow circles represent each subject with C-allele homozygotes and solid circles represent each carriers of the T allele. Mean and $95 \%$ confidence intervals for each group are to the immediate right. DLPFC LICl cluster size has been log transformed and corrected for age (35.5 years). There is significant genotype-by-diagnosis interaction $\left(F_{1.56}=14.3, P=4.1 \times 10^{-4}\right)$. Healthy control C-allele homozygotes $(n=20$; mean: $4.56 \pm 0.52)$ have lower DLPFC LICl cluster size than T-allele carriers $(n=13$; mean $\pm S D$ : $\left.5.08 \pm 0.30 ; F_{1,33}=10.1, P=0.003\right)$. Schizophrenia patients that are $C$-allele homozygotes $(n=10,4.94 \pm 0.34)$ have higher DLPFC LICl cluster size than T-allele carriers $\left(n=13,4.5 \mid \pm 0.53 ; F_{1,23}=4.8, P=0.4\right)$. CNT, healthy control; SCZ, patients with schizophrenia; SD, standard deviation.

in vivo NDMA receptor agonist treatment leads to reduced GAD1 mRNA expression, suggesting upstream control of inhibition (Lisman et al, 2008). GABA $\mathrm{A}_{\mathrm{A}}$ receptor and NMDA receptor abnormalities observed in schizophrenia could explain the opposite effect of GAD1 genotype on LICI cluster size in healthy controls and schizophrenia patients (Akbarian et al, 1995; Uhlhaas and Singer, 2010). However, it is important to be remain cautious about these interpretations given the exploratory nature of the analysis.

Our finding may have clinical implications in the treatment of working memory dysfunction in schizophrenia. Currently, there are no currently approved treatments that are clinically effective, although some preclinical studies have shown effects of brain stimulation. For example, it has been reported that bilateral rTMS treatment to the DLPFC might improve working memory performance in schizophrenia patients (Barr et al, 2013). Also, long-term rTMS treatment was associated with increased prefrontal white matter FA (Allendorfer et al, 2012; Peng et al, 2012). Cortical inhibition has been suggested to explain the lasting effects of rTMS (Daskalakis et al, 2006; Funke and Benali, 2011). Therefore, GAD1 genotype could predict a direct mechanistic link between rTMS treatment and DLPFC LICI. Furthermore, cognitive remediation therapy may ameliorate working memory dysfunction in schizophrenia. Considering our associations among GAD1, prefrontal cortex white matter FA, DLPFC LICI, and working memory, it could be speculated that GAD1 rs3749034 genotype may be an important genetic predictor of adjunctive treatments such as rTMS. GAD1 rs3749034 genotype may be a potential confounder in future treatment trials, and therefore segregating subjects by genotype may improve trial outcome.

This study has a number of limitations. Even though we found a significant association between rs3749034 and schizophrenia, evidence for association between GAD1 common variants and schizophrenia is relatively weak with no reported genome-wide significant findings. However, there is strong evidence for disruption of GAD1 expression in schizophrenia. Our a priori hypothesis was that GAD1 is an important modifier of the disease process, and we selected the rs3749034 based on its known effect on GAD1 expression. That is, we sought to indirectly model for how altered GAD1 expression may lead to neuroanatomical, neurophysiological, and neurocognitive differences. As the minor allele frequency of the rs3749034 variant is relatively low, for statistical analysis we inferred a dominant model by grouping minor allele risk homozygotes and heterozygotes. We limited our analysis only to Caucasian subjects; therefore, we are unable to assess the effect of GAD1 in other ethnic groups. There were differences in the education level of the parents as well as in IQ between groups, which may limit the generalizability for our results. Moreover, the exploratory LICI analysis was performed in a small sample for genetic analysis and subjects were not assessed for working memory performance. Thus, we could not directly assess the relationship among GAD1, LICI, and working memory. Correlations between LICI and working memory has been independently replicated in three healthy control samples (Daskalakis et al, 2008; Hoppenbrouwers et al, 2012; Rogasch et al, 2014). However, this relationship has not been assessed in patients with schizophrenia, and as such our results may help inform molecular or neurophysiologically informed treatment translation efforts to enhance cognitive performance based on GAD1 genotype.

Working memory dysfunction is one of the most intractable symptoms of schizophrenia, with severe consequences on functional outcome (Lett et al, 2014). We have provided evidence that GAD1 may mediate lower working memory function via changes in white matter FA in the prefrontal cortex, and LICI after DLPFC stimulation. Thus, the effects of $G A D 1$ on brain structure and function may explain some of the heterogeneity in working memory dysfunction. In addition, our results suggest a genetic relationship between inhibitory signaling and working memory dysfunction among healthy individuals and schizophrenia patients.

\section{FUNDING AND DISCLOSURE}

Dr Daskalakis has received research and equipment in-kind support for an investigator-initiated study through Brainsway. He has also served on the advisory board for Sunovion, Hoffmann-La Roche Limited, and Merck, as well as received speaker support from Eli Lilly. Dr Walter has received speaker fees from Servier. Dr Kennedy is a Scientific Advisory Board member (unpaid) of AssureRx Health, received speaker honoraria and expenses from Eli Lilly, Novartis, and Shire, and consultant honoraria from Roche. $\mathrm{Dr}$ Mulsant has received medications for NIH-funded 
clinical trials from Bristol-Myers Squibb, Pfizer, and Eli-Lilly. Drs Lett, Radhu, Dominguez, Chakravarty, Nazeri, Farzan, Heinz, and Voineskos report no biomedical financial interest or potential conflict of interest.

\section{ACKNOWLEDGMENTS}

This study is supported by a Canadian Institute of Health Research (CIHR) Fellowship (Dr Lett). Dr Voineskos is supported by the Centre for Addiction and Mental Health (CAMH) Foundation (Kimel Family, Koerner New Scientist Award, and Paul E Garfinkel New Investigator Catalyst Award); Ontario Mental Health Foundation (OMHF); Brain and Behaviour Research Foundation; National Institute of Mental Health. Dr Daskalakis is supported by OMHF, CIHR, the Brain and Behaviour Research Foundation and the Temerty Family and Grant Family, and through the CAMH Foundation and the Campbell Institute. Dr Chakravarty is supported by the BIHR, National Sciences and Engineering Research Council of Canada, Weston Brain Institute, Alzheimer's Society, Michael J Fox Foundation for Parkinson's Research, Brain Canada, and Fonds de Recherche Sante Quebec. Dr Nazeri is supported by a CAMH Fellowship. Dr Radhu is supported by an OMHF studentship.

\section{REFERENCES}

Aarts E, Roelofs A, Franke B, Rijpkema M, Fernandez G, Helmich RC et al (2010). Striatal dopamine mediates the interface between motivational and cognitive control in humans: evidence from genetic imaging. Neuropsychopharmacology 35: 1943-1951.

Akbarian S, Huntsman MM, Kim JJ, Tafazzoli A, Potkin SG, Bunney WE Jr et al (1995). GABAA receptor subunit gene expression in human prefrontal cortex: comparison of schizophrenics and controls. Cerebral cortex 5: 550-560.

Allendorfer JB, Storrs JM, Szaflarski JP (2012). Changes in white matter integrity follow excitatory rTMS treatment of post-stroke aphasia. Restor Neurol Neurosci 30: 103-113.

Barr MS, Farzan F, Rajji TK, Voineskos AN, Blumberger DM, Arenovich $\mathrm{T}$ et al (2013). Can repetitive magnetic stimulation improve cognition in schizophrenia? Pilot data from a randomized controlled trial. Biol Psychiatry 73: 510-517.

Ben-Ari Y, Khalilov I, Kahle KT, Cherubini E (2012). The GABA excitatory/inhibitory shift in brain maturation and neurological disorders. The Neuroscientist 18: 467-486.

Brauns S, Gollub RL, Walton E, Hass J, Smolka MN, White T et al (2013). Genetic variation in GAD1 is associated with cortical thickness in the parahippocampal gyrus. J Psychiatr Res 47: 872-879.

Daskalakis ZJ, Farzan F, Barr MS, Rusjan PM, Favalli G, Levinson AJ et al (2008). Evaluating the relationship between long interval cortical inhibition, working memory and gamma band activity in the dorsolateral prefrontal cortex. Clin EEG Neurosci 39: 150-155.

Daskalakis ZJ, Moller B, Christensen BK, Fitzgerald PB, Gunraj C, Chen R (2006). The effects of repetitive transcranial magnetic stimulation on cortical inhibition in healthy human subjects. Exp Brain Res 174: 403-412.

Deisz R (1999). GABA B receptor-mediated effects in human and rat neocortical neurones in vitro. Neuropharmacology 38: $1755-1766$.

Farrant M, Nusser Z (2005). Variations on an inhibitory theme: phasic and tonic activation of GABA(A) receptors. Nat Rev Neurosci 6: 215-229.
First MB, Spitzer RL M, Gibbon M, JBW Williams (1995). Strucutured Clinical Interview for DSM-IV Axis I Disorders, Patient Edition (SCID-P), Version 2. Biometrics Research: New York, NY.

Folstein MF, Folstein SE, McHugh PR (1975). 'Mini-mental state'. A practical method for grading the cognitive state of patients for the clinician. Nat Rev Neurosci 12: 189-198.

Funke K, Benali A (2011). Modulation of cortical inhibition by rTMS-findings obtained from animal models. J Physiol 589: 4423-4435.

Garcia Dominguez L, Radhu N, Farzan F, Daskalakis ZJ (2014). Characterizing long interval cortical inhibition over the timefrequency domain. PLoS One 9: e92354.

Gibbs RA, Belmont JW, Hardenbol P, Willis TD, Yu F, Yang $\mathrm{H}$ et al (2003). The international HapMap project. Nature 426: 789-796.

Green AE, Kraemer DJ, Deyoung CG, Fossella JA, Gray JR (2013). A gene-brain-cognition pathway: prefrontal activity mediates the effect of COMT on cognitive control and IQ. Cerebr Cortex 23: 552-559.

Greenwood TA, Braff DL, Light GA, Cadenhead KS, Calkins ME, Dobie DJ et al (2007). Initial heritability analyses of endophenotypic measures for schizophrenia: the consortium on the genetics of schizophrenia. Archiv Gen Psychiatry 64: 1242-1250.

Holdnack HA (2001). Wechsler test of adult reading: WTAR. The Psychological Corporation: San Antonio, TX.

Hopfinger JB, Buonocore MH, Mangun GR (2000). The neural mechanisms of top-down attentional control. Nature Neurosci 3: 284-291.

Hoppenbrouwers SS, De Jesus DR, Stirpe T, Fitzgerald PB, Voineskos AN, Schutter DJLG et al (2012). Inhibitory deficits in the dorsolateral prefrontal cortex in psychopathic offenders. Cortex 49: 1377-1385.

Hyde TM, Lipska BK, Ali T, Mathew SV, Law AJ, Metitiri OE et al (2011). Expression of GABA signaling molecules KCC2, NKCC1, and GAD1 in cortical development and schizophrenia. J Neurosci 31: 11088-11095.

Kanai R, Rees G (2011). The structural basis of inter-individual differences in human behaviour and cognition. Nat Rev Neurosci 12: 231-242.

Keel JC, Smith MJ, Wassermann EM (2001). A safety screening questionnaire for transcranial magnetic stimulation. Clin Neurophysiol 112: 720.

Klumpers F, Kroes MC, Heitland I, Everaerd D, Akkermans SE, Oosting RS et al (2014). Dorsomedial prefrontal cortex mediates the impact of serotonin transporter linked polymorphic region genotype on anticipatory threat reactions. Biol Psychiatry 78: 582-589.

Kochunov P, Jahanshad N, Sprooten E, Nichols TE, Mandl RC, Almasy L et al (2014). Multi-site study of additive genetic effects on fractional anisotropy of cerebral white matter: comparing meta and megaanalytical approaches for data pooling. Neuroimage 95: 136-150.

Lazarus MS, Krishnan K, Huang ZJ (2013). GAD67 deficiency in parvalbumin interneurons produces deficits in inhibitory transmission and network disinhibition in mouse prefrontal cortex. Cerebr Cortex 25: 1290-1296.

Lett TA, Voineskos AN, Kennedy JL, Levine B, Daskalakis ZJ (2014). Treating working memory deficits in schizophrenia: a review of the neurobiology. Biol Psychiatry 75: 361-370.

Lewis DA, Curley AA, Glausier JR, Volk DW (2012). Cortical parvalbumin interneurons and cognitive dysfunction in schizophrenia. Trends Neurosci 35: 57-67.

Lewis DA, Hashimoto T, Volk DW (2005). Cortical inhibitory neurons and schizophrenia. Nat Rev Neurosci 6: 312-324.

Lisman JE, Coyle JT, Green RW, Javitt DC, Benes FM, Heckers S et al (2008). Circuit-based framework for understanding neurotransmitter and risk gene interactions in schizophrenia. Trends Neurosci 31: 234-242. 
Maris E, Oostenveld R (2007). Nonparametric statistical testing of EEG- and MEG-data. J Neurosci Methods 164: 177-190.

McClintock SM, Freitas C, Oberman L, Lisanby SH, Pascual-Leone A (2011). Transcranial magnetic stimulation: a neuroscientific probe of cortical function in schizophrenia. Biol Psychiatry 70: 19-27.

McDonnell MN, Orekhov Y, Ziemann U (2006). The role of GABA (B) receptors in intracortical inhibition in the human motor cortex. Exp Brain Res 173: 86-93.

Miller MD, Paradis CF, Houck PR, Mazumdar S, Stack JA, Rifai AH et al (1992). Rating chronic medical illness burden in geropsychiatric practice and research: application of the Cumulative Illness Rating Scale. Psychiatry Res 41: 237-248.

Patel AB, de Graaf RA, Martin DL, Battaglioli G, Behar KL (2006). Evidence that GAD65 mediates increased GABA synthesis during intense neuronal activity in vivo. J Neurochem 97: 385-396.

Peng H, Zheng H, Li L, Liu J, Zhang Y, Shan B et al (2012). Highfrequency rTMS treatment increases white matter FA in the left middle frontal gyrus in young patients with treatment-resistant depression. J Affect Disord 136: 249-257.

Pirkola T, Tuulio-Henriksson A, Glahn D, Kieseppa T, Haukka J, Kaprio J et al (2005). Spatial working memory function in twins with schizophrenia and bipolar disorder. Biol Psychiatry 58: 930-936.

Radhu N, Garcia Dominguez L, Farzan F, Richter MA, Semeralul MO, Chen $\mathrm{R}$ et al (2015). Evidence for inhibitory deficits in the prefrontal cortex in schizophrenia. Brain 138: 483-497.

Rogasch NC, Daskalakis ZJ, Fitzgerald PB (2014). Cortical inhibition of distinct mechanisms in the dorsolateral prefrontal cortex is related to working memory performance: a TMS-EEG study. Cortex 64C: 68-77.

Rossi S, Hallett M, Rossini PM, Pascual-Leone A, Safety of TMSCG (2009). Safety, ethical considerations, and application guidelines for the use of transcranial magnetic stimulation in clinical practice and research. Clin Neurophysiol 120: 2008-2039.

Schizophrenia Working Group of the Psychiatric Genomics Consortium (2014). Biological insights from 108 schizophreniaassociated genetic loci. Nature 511: 421-427.

Straub RE, Lipska BK, Egan MF, Goldberg TE, Callicott JH, Mayhew MB et al (2007). Allelic variation in GAD1 (GAD67) is associated with schizophrenia and influences cortical function and gene expression. Mol Psychiatry 12: 854-869.

Thompson PM, Cannon TD, Narr KL, van Erp T, Poutanen VP, Huttunen $\mathrm{M}$ et al (2001). Genetic influences on brain structure. Nat Neurosci 4: 1253-1258.

Torrey EF, Barci BM, Webster MJ, Bartko JJ, Meador-Woodruff JH, Knable MB (2005). Neurochemical markers for schizophrenia, bipolar disorder, and major depression in postmortem brains. Biol Psychiatry 57: 252-260.

Uhlhaas PJ, Singer W (2010). Abnormal neural oscillations and synchrony in schizophrenia. Nat Rev Neurosci 11: 100-113.

Voineskos AN, Rajji TK, Lobaugh NJ, Miranda D, Shenton ME, Kennedy JL et al (2012). Age-related decline in white matter tract integrity and cognitive performance: a DTI tractography and structural equation modeling study. Neurobiol Aging 33: 21-34.

Supplementary Information accompanies the paper on the Neuropsychopharmacology website (http://www.nature.com/npp) 